\title{
CHANGES IN PERIPHERAL LEUKOCYTE AND BODY FLUIDS OF ONCHOCERCIASIS PATIENTS TREATED WITH IVERMECTIN
}

\author{
Manafa, O. U., Mafe, M. A., Idowu, E. T., Ajayi, M. B. \\ Nigerian Institute of Medical Research \\ 6, Edmond Crescent, PMB, 2013, Yaba, Lagos
}

Correspondence to: 0 . U. Manafa (ogennam@yahoo.com)

\begin{abstract}
This study evaluated the peripheral leukocyte count and the presence of microfilariae in the body nuids of onchocerciasis patients treated with ivermectin. Fifty-three patients over the age 10 years were selected from Ipogun, an onchocerciasis endemic area in Ondo state, Migeria. Before and after treatment, all patients recelved a parasitological and clinical examination that included physical examination, palpation of onchocercal nodules, assessment of microfilarial densities in iliac crest slin snips, diagnosis of concomitant parasitic infections in stool specimens and total leukocytes differential counts. Results indicated that ivermectin did not induce a decrease in the total number of peripheral leukocytes but there was a decrease in the number of eosinophils. Illerofilariae were not found in increase frequency in the urine, blood and aputum, while the number of microfilariae per ms of akin snip decreaned.
\end{abstract}

\section{INTRODUCTION}

Onchocerciasis is a major health problem in Nigeria. Epidemiological studies have shown that some communities are severely affected by the disease both in the Northern regions and in the Southern forested areas with foci of blinding disease found in the Northern parts of the country. Drug treatment and control of onchocerciasis have been unsatisfactory in the past. The available drug diethylcarbamazine (DEC) and suramin are too toxic for mass distribution.

The introduction of drug ivermectin has been responsible for the most recent dramatic advances both in disease control $(1,2)$ and in interruption of transmission $(3$, 4). Ivermectin has replaced DEC, and community based mass treatment campaigns against Onchocerca volvulus were initiated by the Onchocerciasis Control Program (OCP) in West Africa. Ivermectin is believed to paralyze susceptible nematodes by affecting neurotransmission mediated by gamma-amino-N-butyric acid (5), but total immobilization or killing of microfilariae in vivo has never been observed (6) and the exact mode of antifilarial action. remain unclear.

Observations also indicate that ivermectin might not act on the filarial parasite directly, but rather, in synergism with the host immune response (7). Since ivermectin reduces microfilariae of Onchocerca volvulus and the drug acts in synergism with the host's immune response, it is expected that the drug would affect the clinical and systemic as well as immunological balance of the host.

The present study was designed to examine the eosinophilic leukocytic reactions to ivermectin provocation since filarial infections are usually followed by eosinophilia. It is also intended to correlate the signs and symptoms of the clinical response and the alterations in numbers of microfilariae in body fluids associated with a single oral dose of ivermectin in population of people with moderate to heavy infection with Onchocerca volvulus. 
MATERTALS AND METHOD

Patient population and evaluation

The study" was carried out in Ipogun, a town in Ifedore Local Government Area in Ondo State, Nigeria. Previous data from the study in the area showed that onchocerciasis is hyper endemic with a prevalence rate of $34 \%$ for leopard skin, and $16 \%$ nodular rate $(8)$ which is representative of forest type disease. Ipogun is a rural community with a population of about 2000 residents.

Fifty three residents over the age of 10 years comprised the study patients. Children less than 10 years old, debilitated individuals, pregnant women and lactating mothers were excluded from the study. Patients were evaluated clinically and immunologically immediately before and after repeated $150 \mu \mathrm{g} / \mathrm{kg}$ doses of ivermectin given annually for 2 years. Samples (skin, blood, urine, and sputum) were collected before treatment, 2 days, 3 days, 3 months, 6 months, 12 months and 18 months after treatment. The patients co-operated all through in all aspects of the study except the blood sample at 48 hours and 72 hours. Clinical evaluation included physical examination, specific examination for the presence of onchocercal nodules, assessment of microfilarial densities in iliac crest skin snips and body fluids, total leukocyte and differential counts. All the subjects evaluated met the following criteria; positive history of exposure in endemic regions, clinical symptoms consistent with onchocerciasis and positive skin snips.

Skin snips were taken with corneoscleral punch from both iliac crests and placed in wells of microtitre plates containing physiological saline. Emergent microfilariae was counted immediately and 24 hours after. The number of microfilariae were counted and scored quantitatively as reported by Crosskey and Crosskey (9).

Total and differential white blood cell count

Blood was anticoagulated with 1 $\mathrm{mg} / \mathrm{ml}$ ethylene diamine tetra acetate (EDTA) and white blood cell count was done by conventional methods. Blood eosinophil count was done by the method of Discombe (10). Differential white blood cell count was done on smears stained with Leishmans stains.

\section{Body fluid examination}

The sediments of urine and blood specimens were treated according to previous methods $(11,12)$ and were checked for microfilaria of Onchocerca volvulus. Each sputum specimen was preserved with tincture of Merthiolate and examined similarly like the urine specimens.

\section{Statistical analysis}

Differences in means between time points were compared using student's test on logarithmically transformed data.

\section{RESULTS}

\section{Pretreatment findings}

Fifty three individuals aged $15-72$ years (mean age 41.2 years) were evaluated before treatment. All were microfiladermic (geometric mean 22.72, 1-204/mg of skin) and 8 had detectable subcutaneous nodules. Thirty two of them were positive for leopard skin while ocular examination showed normal visual acuity except for 7 persons who complained of blurred vision (Table 1).

\section{Complications during treatment}

Fourteen patients $(26.4 \%)$ had moderate side effects. Two patients experienced severe adverse reactions, of 
which they received additional treatment during the follow up period of 3 days.

skin microfilarial levels

Levels of microfilaraia, assessed by skin snips just before ivermectin administration, were measured at 6 months interval. There was a significant reduction $(p<0.05)$ in the microfilarial level 6 months after the 1 st dose of ivermectin (Table 2). Of the 53 patients, $22(41.5 \%)$ had no skin microfilariae at the final sampling.

\section{Total Leukocyte and Eosinophil counts}

Ivermectin therapy had no effect on the total leukocyte counts. There was no significant difference at either the population or individual level between the pretreatment values and any of the subsequent time points (Table 2). In contrast; there was a rise in the absolute eosinophil counts two and three days after treatment. But by the third month after

Table 1: Baseline characteristics of patients treated with ivermectin

No of patients

53

Mean age (Range in $\mathrm{kg}$ )

41.2(15-72)

Mean weight (Range in $\mathrm{Kg}$ )

$59(34-84)$

No of patients with

1. Onchocerciasis 34

2. $\because$ Leopard skin 30

3. $\therefore$ Nodules 8

4. Blurred vision 7 treatment, there was a considerable fall in the eosinophil count reaching statistical significance levels by 12 months No correlation was seen between the reduction in eosinophil counts and the decrease in skin-microfilarial levels, nor was there a correlation between eosinophil level and skin microfilarial density pretreatment.

Microfilariae levels in the body fluids

No onchocercal microfilaria was found in the urine, blood and sputum of patients before and after treatment. It is worthy to note that in 7 patients with concomitant Schistosoma haematobium infection, ivermectin was found not to have any effect $S$. haematobium ova was found in higher number in 2 cases of patients after treatment, while the number of $S$. haematobium ova remained the same in the other 5 patients before and after treatment. 


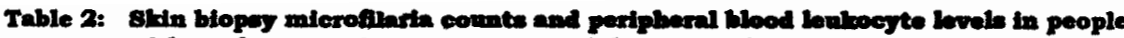

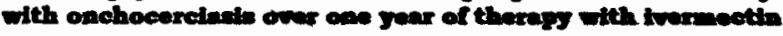

Parmeneter

Mean no of

MF/skin biopay

2ans

30

22.72

7662.4

13.6

4.5

Leukocytes

Peripheral Blood

Eosinophils
Betore trencunent

$$
21.5
$$

$8941.6 \quad 7936$

$18.4 \quad 19.6$
Sinthe

\section{Discussion}

As expected from past studies (1, 13), levels of skin microfilariae decreased significantly during this period. Hematological assessment revealed no change in the leukocyte counts. Eosinophil numbers decreased significantly over the 18 months repeated observations. Such findings are particularly interesting since eosinophils have been known to be causally involved not only in the cytotoxicity to microfilariae after treatment (14) but also in the post treatment Mazzotti reactions (15) and the development of skin pathology (16).

Whether the decrease in blood eosinophil is the result of the hosts decreased antigen load (reduction in microfilariae) or whether if reflects some other possible treatment change in the host is unclear. By whatever mechanism, however, there was a reduction in eosinophil levels in these patients to less than half the pretreatment level. Within 2days after ivermectin was administrated, there was a rise in eosinophil and it has previously been demonstrated that there is a rise in eosinophil levels within the first month after treatment with either ivermectin or DEC (17). With ivermectin therapy the rise occurs more slowly than after DEC treatment, presumably because of a different mechanism of action (18) that results in

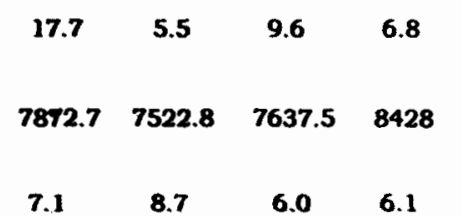

decrease or clearance of the skin microfilariae.

The present finding indicate that after this initial early post treatment eosinophilia, there is a continual decline in eosinophil levels with repeated ivermectin treatment, suggesting that the patients' may be moving towards a normal (homoeostatic) state (19). The present study also demonstrated the effect of a single oral dose of ivermectin on migration of microfilariae in patients with a relatively heavy dose of infection on the skin. After administration of ivermectin the skin snips count tended to decrease with time while microfilariae were not found in the sputum, urine and blood as reported after DEC treatment by (20). This finding agrees with that of Richards et al (21) who also observed that the fall in microfilariae skip concentration after ivermectin treatment was not accompanied by any marked wave of microfilariae in the blood or urine.

The observations of Awadzi et al (17), Richards et al (21) and Basset et al (22) suggest that, after ivermectin administration although some microfilariae may enter the blood stream (presumably by way of the lymphatic system), their numbers are in no way comparable to those seen after DEC. Duke (23) also reported that ivermectinaffected microfilariae may be destroyed in 
the lymph nodes and elsewhere more easily and with less reaction than those unmasked by DEC and fewer of them may survive the lymph node network to pass into the blood. They also observed that ivermectin caused microfilariae to move from the subepidermal layer into the deeper layers of the epidermis, subcutaneous fat, connective tissue and the regional lymph nodes. They concluded that the migration of microfilariae from superficial layers of the skin to the deeper connective tissues, fat lymph nodes, coupled with the mild cellular reactions that surround microfilariae dying from the effects on ivermectin are the main reasons why this effective microfilaricide for the control of onchocerciasis appears to be a promising chemotherapeutic strategy, particularly since it is not associated with severe side effects characteristic of DEC therapy.

Findings in this study demonstrated no increased symptoms that would preclude wide use of ivermectin to treat populations infected with generalized onchocerciasis.

\section{REFERENCES}

1. Aziz AM, Diallo S, Diop IM, Lariviere M, Porta M. Efficacy and tolerance of ivermectin in human onchocerciasis. Lancet. 1989; ii: $171-173$

2. Pacque M, Munoz B, Greene BM, White AT, Dukuly $Z$, Taylor HR. Safety and compliance with community based ivermectin therapy. Lancet. 1990; 335: 1377-1380.

3. Taylor HR, Pacque M, Minoz B, Greene BM. Impact of mass treatment of onchocerciasis with ivermectin on the transmission of infection. Science. 1990; 250: $116-118$

4. Trpis M, Childs JE, Fryyauff DJ, et al. Effect of mass treatment of a human population with ivermectin on transmission of Onchocerca volvulus by Simulium yahanse in Liberia, West Africa. Am. J. Trop. Med. Hyg.1990; 42: 148-156.

5. Holden-Dye L, Walker RJ. Avermectin and avermectin derivatives are antagonist at the 4-aminobutyric acid (GABA) receptor on the somatic muscle cells of Ascaris. Parasitology. 1990; 101: 265-271.
6. Jurgens S, Schultz-Key H. Effect of ivermectin on the vertical distribution of Onchocerca volvulus microfilariae in the skin. Trop. Med. Parasitol. 1990; 14: 165-168.

7. Schultz-Key $H$. Ivermectin in the treatment of onchocerciasis. In: ISI Atlas of Science: Pharmacology. Institute for Scientific Information, Philadelphia. 1987: 246-249

8. Federal Ministry of Health Report on rapid assessment method for community diagnosis of onchocerciasis in Ondo State, Nigeria. 1994: 100

9. Crosskey RW, Crosskey ME. A quantitative survey of onchocerciasis in persons under 20 years in an endemic area in Notthern Nigeria. Ann. Med. Parasitol. 1959; 32: 2-16.

10. Discombe E. Criteria of eosinophilia. Lancet. 1946; 1: 195

11. Fazan LE, Anderson RI, Marroquin HF. Clinical and laboratory changes consequent to diethylcarbamazine inpatients with onchocerciasis. Am. J. Trop. Med. Hyg. 1976; 25: 250-255

12. Anderson J, Fulsang H, Hamilton PJS, Marshall TF. The prognostic value of head nodules and microfilariae in the skin in relation to ocular onchocerciasis. Tropenmed Und Parasitol. 1975; 26: 160 166

13. Lariviere $\mathrm{M}$, Beauvias $\mathrm{B}$, Aziz $\mathrm{EM}$, et al Etude en Cote-d'ivoire (1985-1987) de l'efficacite Et de la tolerance de' ivermectin (Mectizan) dans 1' Onchocercose humaine. Bull. Soc. Pathol. Exot. Filiales. 1987; 82: 35-47

14. Mackenzie CD, Williams JF, Sisley BM, Steward MW, O'Day J. Variations in host responses and the pathogenesis of human onchocerciasis. Rev. Infect. Dis. 1985; 7: 802-808

15. Francis H, Awadzi K, Ottesen EA. The Mazzotti reaction following treatment of onchocerciasis with diethylcarbamazine: clinical severity as a function of infection intensity. Am. J. Trop. Med. Hyg. 1985; 4: $529-536$

16. Conner DH, George GH, Gibson DW. Pathologic changes of human onchocerciasis. Rev. Infect. Dis. 1985; 7: 802-808

17. Awadzi K, Dadzie KT, Schultz-Key H. The chemotherapy of onchocerciasis $\mathbf{x}$, an assessment of four single dose treatment regimens of $\mathrm{mk}-933$ (ivermectin) in human onchocerciasis. Ann. Trop. Med. Parasitol. 1986; 79: 63-78

18. Soboslay PT, Newland HS, White AT, et al. Ivermectin effect on microfilariae of Onchocerca volvulus after a single oral dose in humans. Trop. Med. Parasitol. 1987; 38: 8-10

19. Cathy Steel, Lujan-Tragay A, GonzalezPeraita C, Zea-Flores G, Nutman TB. Immunologic responses to repeated ivermectin treatment in patients with 
onchocerciasis J. Infect. Dis. 1991; 164: 581-587

20. * Duke BOL, Moore PJ, Vincelette J. The population dynamics of Onchocerca volvulus during treatment with suramin and diethylcarbamazine. Tropenmed. Parasitol. 1976; 27:133-144

21. Richards FO, Zea-Flores R, Duke BOL. Dynamics of microfilariae of Onchocerciasis volvulus over the first 72 hours after treatment with ivermectin. Trop. Med. Parasitol. 1989; 40: 299-303
22. Basset DP, Basset $\mathrm{A}$, Larivivere $\mathbf{M}$. Effects de la diethylcarbamazine Et de L'ivermectine sur la mobilization des microfilaires d' Onchocerciasis volvulus. Path. Biol. 1989; 37: 668-672

23. Duke BOL, Soula L, Zea-Flores G, Braatthauer GL, Doumbo $O$. Migration and death of skin dwelling microfilariae after treatment with ivermectin. Tropenmed. Parasitol 1991; 42: 25-30 